\title{
Promouvoir et diffuser la culture scientifique
}

Commission "Culture scientifique » de la Société Française de Physique

Partie intégrante de la

Culture, la culture scientifique

n'est pas toujours présente

à l'esprit lorsque l'on parle de

" culture générale ». Elle aussi

pourtant s'acquiert, s'entretient

et se diffuse au bénéfice

des mêmes deux publics :

les jeunes et les « citoyens »

adultes.

Le texte qui suit a pour objet

de souligner le rôle des acteurs

chargés de la transmettre,

intermédiaires nécessaires

entre ces publics et les scien-

tifiques "producteurs » de

connaissances. Aux médiateurs

et aux professionnels des

sciences et techniques,

de mettre en commun leurs

compétences pour transmettre

des savoirs sous des formes

accessibles.
Il y a un siècle, en France, les personnes cultivées, c'est-à-dire ayant au moins poursuivi leurs études jusqu'au baccalauréat, possédaient des notions sérieuses non seulement en lettres et en arts, mais également en sciences. Pour s'en convaincre, il suffit de consulter un manuel de l'époque, par exemple un traité de physique élémentaire pour les classes de philosophie, programme de 1912 [1]. On peut être stupéfait. Ce n'est malheureusement plus le cas aujourd'hui. Pourtant les sciences et les techniques jouent un rôle majeur dans le monde actuel : " on croit pouvoir être cultivé, honnête homme en quelque sorte, en faisant mine d'ignorer ce qui conditionne de plus en plus la vie [2]".

La culture scientifique n'est pas seulement un minimum de connaissances, une somme de savoirs, mais elle est aussi une certaine compréhension de la démarche scientifique. Celle-ci part de l'observation et de l'expérimentation, avant d'arriver à une interprétation et une généralisation conduites avec esprit critique. Cet avancement de la science ne va pas sans le doute, sans débats et parfois sans remises en cause ; l'histoire des sciences nous permet d'en saisir la progression qui n'est pas toujours linéaire.

\section{La culture scientifique à l'école}

Les futurs citoyens sont supposés acquérir une culture scientifique au cours de leurs scolarités primaire et secondaire.

L'enseignement primaire se doit de développer la curiosité, l'esprit d'observation et des rudiments d'expérimentation chez les élèves. Si le cycle CP-CE1 est consacré aux mathématiques et à la découverte du monde, le programme du cycle des approfondissements (CE2-CM1-CM2) est en partie dévolu à un développement de la culture scientifique des élèves [3]. Les programmes de collège [4] et lycée insistent de la même manière sur l'acquisition d'une culture scientifique. L'enseignement secondaire doit apporter à tous un certain nombre de connaissances de base et un aperçu de la démarche scientifique, via un enseignement expérimental nécessaire. C'est dans cet esprit que les sciences devraient être enseignées à tous les élèves et pas seulement à ceux qui se destinent aux sciences et techniques.

L'acquisition de la culture scientifique à l'école a l'enseignant pour principal vecteur. En pratique la situation n'est pas aussi simple : le peu de formation scientifique, y compris de formation continuée, de la majorité des enseignants du primaire leur permet difficilement d'effectuer une véritable initiation des élèves aux sciences. Les efforts faits à l'initiative de "La Main à la pâte " [5], ou sur la base de la pédagogie Freinet [6], restent insuffisants car ils touchent in fine peu d'élèves. Un enseignant non scientifique peut actuellement faire appel à ses collègues ou à un intervenant extérieur venant du milieu associatif, d'un organisme de médiation, ou de la recherche. Les CCSTI [7] et diverses associations, voire même des universités, disposent souvent du matériel et des compétences pour ce genre d'intervention. Mais il faut souligner le caractère ponctuel de ces actions et le fait qu'elles laissent peu de traces si elles n'ont pas été préparées en classe et approfondies par l'enseignant. Les musées des sciences et techniques peuvent aussi jouer un rôle important.

Dans le secondaire, les programmes privilégient actuellement l'accumulation des connaissances par rapport à l'acquisition de la démarche scientifique. D'autre part, depuis déjà quelque temps le "formel » a pris le pas sur la présentation pragmatique des faits, suivie de leur interprétation. En outre, la baisse importante des heures d'enseignement expérimental dans le secondaire est très dommageable. Le triptyque savoir/ 

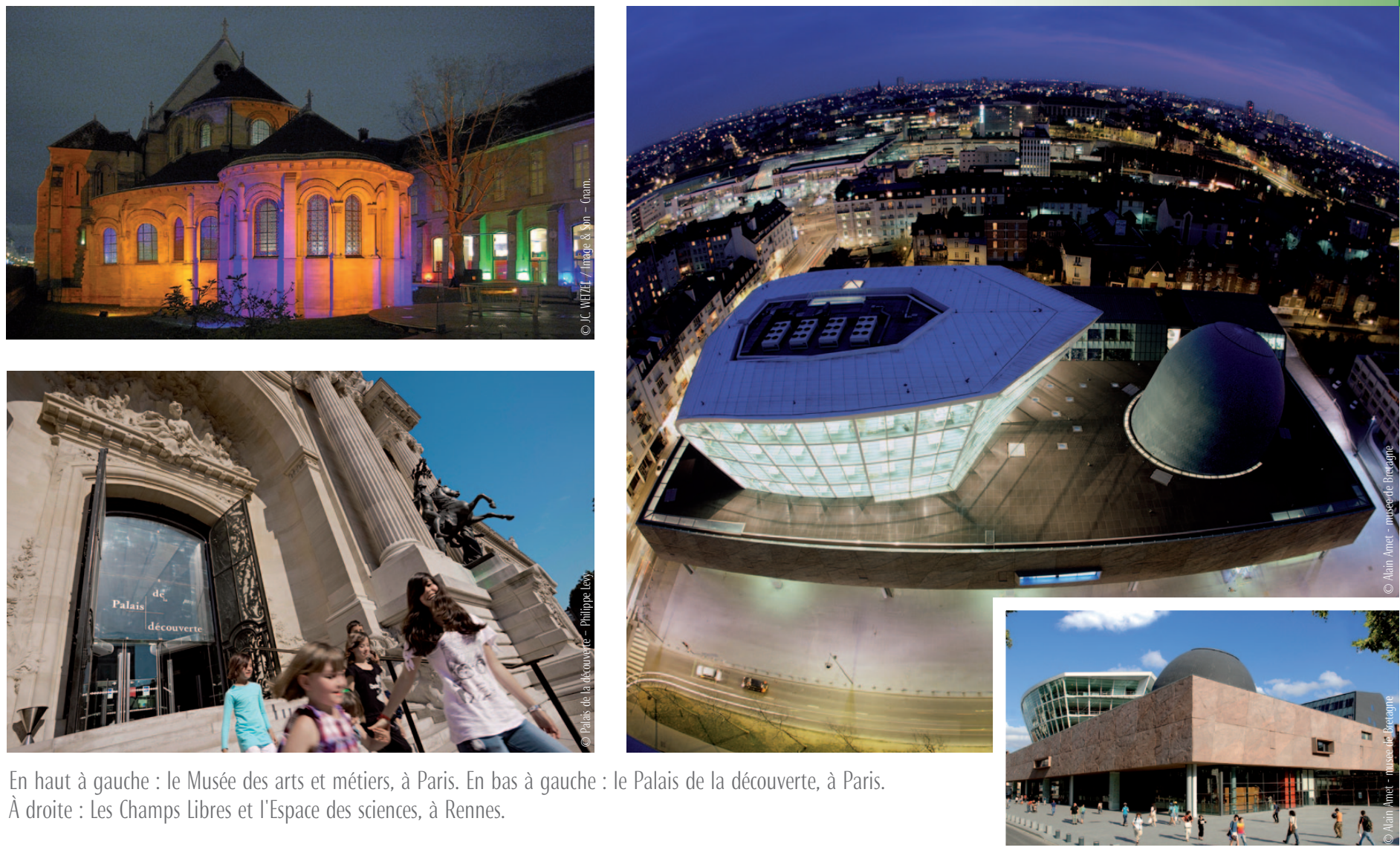

En haut à gauche : le Musée des arts et métiers, à Paris. En bas à gauche : le Palais de la découverte, à Paris. À droite : Les Champs Libres et l'Espace des sciences, à Rennes.

raisonnement/expérimentation est actuellement très déséquilibré.

La formation initiale et continue des professeurs devrait être revue à tous les niveaux, sans omettre l'indispensable formation pédagogique, très délaissée actuellement.

\section{La culture scientifique pour le grand public}

Comme on l'a souligné plus haut, et c'est vrai partout dans le monde, la «culture générale » n'implique plus spontanément sa composante scientifique ; de plus, surtout en France, la formation de base des décideurs ne comprend que peu de culture scientifique.

La culture scientifique moyenne d'un bachelier est actuellement insuffisante dans une société où les progrès scientifiques et technologiques rapides sont souvent sujets d'inquiétude. Ceci souligne encore le besoin d'une diffusion forte de la culture scientifique dans l'ensemble de la société. Le succès d'audience assez général des conférences grand public, bars des sciences, universités de tous les savoirs, fêtes de la science, est un bon indicateur du besoin ressenti par le "grand public ».

Les normes démocratiques exigent que la société intervienne dans les choix technologiques au cours de débats ouverts et documentés, ce qui entraîne la nécessité pour tout citoyen d'avoir une connaissance scientifique minimale. Si le critère scientifique ne doit pas être considéré comme prioritaire dans les choix démocratiques, car il est insuffisant, il est cependant nécessaire pour comprendre les enjeux de tel ou tel choix technologique, qu'il porte par exemple sur la bioéthique ou sur la production et la consommation d'énergie. En outre, des abus industriels rendent les progrès scientifiques et techniques suspects aux yeux d'une grande partie de la population. Certains "progrès ", imposés pour des motifs financiers (médicaments, OGM, etc), sans que leurs bienfaits soient avérés, doivent être compris avant d'être dénoncés.

La complexité et la spécialisation de plus en plus pointue dans la science moderne comme dans la technologie, deviennent un handicap pour la transmission efficace des savoirs. Le scientifique ne dispose en général ni du temps ni des techniques de communication nécessaires. Le besoin de médiateurs entre les scientifiques et le " grand public " est de plus en plus indispensable : lorsqu'un sujet de société comporte un versant scientifique, celui-ci est souvent " expédié "; seul un médiateur ayant des connaissances précises sur le sujet et des qualités de synthèse peut alors faire œuvre utile. Ce médiateur ne peut être que quelqu'un de rompu à la communication scientifique.
Les CCSTI, de création récente, et diverses associations regroupent de tels spécialistes et animent la médiation de façon souvent remarquable. Si la part de bénévolat est toujours importante, la médiation s'est professionnalisée dans les vingt dernières années.

Les médias devraient jouer un rôle moteur dans la diffusion de la culture scientifique. Il faut souligner la qualité d'émissions de radio comme "La tête au carré " (Mathieu Vidard, son animateur, vient d'ailleurs de se voir attribuer le prix Jean Perrin de la SFP), et comme "La marche des sciences " d'Aurélie Luneau, en particulier sur les antennes de RadioFrance, celle d'émissions télévisées comme «C'est pas sorcier » ou « $\mathrm{E}=\mathrm{mc}^{2}$ » sur France-Télévision. Une information scientifique de qualité est aussi disponible dans des revues spécialisées comme La Recherche, Pour la Science ou Sciences et Avenir et divers livres ou des sites dédiés, comme ceux de l'Académie des sciences, du CNRS, du CEA et des sociétés savantes. Par contre, rares sont les articles véritablement documentés dans la presse écrite généraliste (quotidiens ou hebdomadaires) : on préfére y donner la parole au sensationnel ou aux angoisses (climat, ondes électromagnétiques, nucléaire), sans favoriser une controverse véritablement scientifique. Il est vital que les responsables 
$>>$

des médias prennent de nouveau en compte le fait qu'une information scientifique de qualité ne peut être produite, que par un journaliste dont la formation et les contacts réguliers avec les milieux scientifiques en garantissent le sérieux. La quasi-disparition, en France comme dans plusieurs pays étrangers, de la profession de journaliste scientifique est à la fois une cause et un révélateur. Une formation aux sciences et aux techniques devrait donc être introduite d'urgence dans les écoles de journalisme.

Enfin, les citoyens dans leur ensemble ont besoin de dirigeants et d'élus munis des connaissances scientifiques de base leur permettant de prendre en connaissance de cause des décisions essentielles à caractère technologique, souvent annonciatrices d'évolutions sociétales. À noter que si l'intervention des pouvoirs publics dans la sphère scientifique est évidemment visible via le ministère de l'Éducation nationale et celui de l'Enseignement supérieur et de la Recherche, son impact dans la promotion et la diffusion de la culture scientifique reste très discret.

La Société Française de Physique se doit d'apporter sa contribution dans ce secteur, en coopération avec tous les acteurs naturels de la diffusion et de la promotion de la culture scientifique. De nombreux membres de la SFP, en activité ou en retraite, participent à cet effort dans leur région. D'autres aimeraient s'y associer. Une réflexion puis une démarche vers les médias doivent être menées.

Dans cette dynamique, une journée sera conjointement organisée par la SFP et l'AJSPI (Association des journalistes scientifiques de la presse d'information) sur le thème "Science et médias : mieux travailler ensemble ", le lundi 9 janvier 2012, au Palais de la découverte.

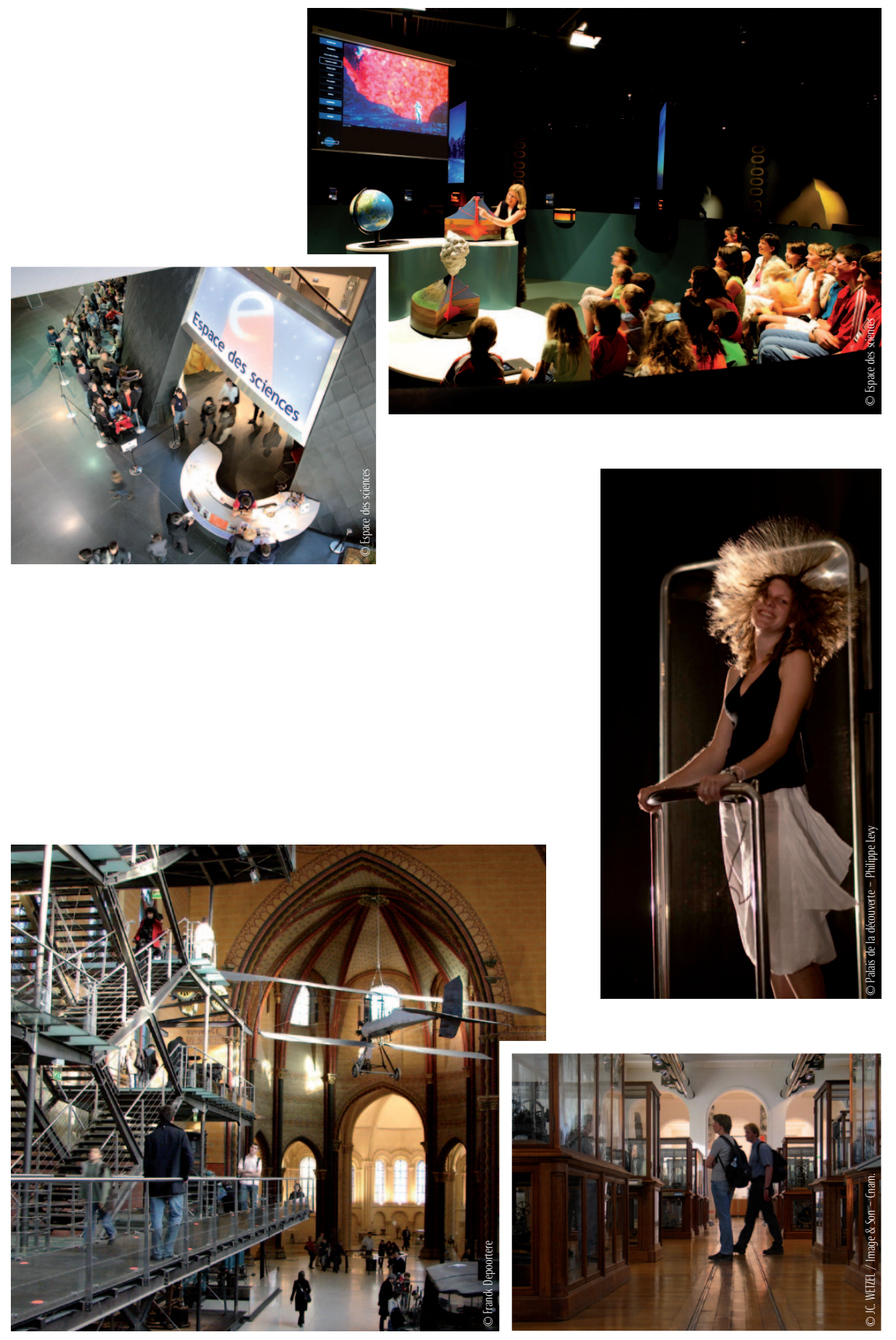

Observation, questionnement, expérimentation et argumentation pratiqués sont essentiels pour atteindre ces buts. Un exemple : l'esprit de la Main à la pâte. C'est pourquoi les connaissances et les compétences sont acquises dans le cadre d'une démarche d'investigation. Ces recherches développent la curiosité, la créativité, l'esprit critique et l'intérêt pour le progrès scientifique et technique. "

4 - www.education.gouv.fr/cid81/les-programmes. html\#Physique\%20-\%20chimie

Ainsi, l'objectif du programme de physique-chimie du collège énonce :

"Acquérir les bases d'une culture scientifique dans différents domaines de la physique et de la chimie.
En s'appuyant avant tout sur l'expérimentation, les élèves acquièrent les premières notions scientifiques sur la matière, la lumière, l'électricité, la gravitation. Ils développent leur sens de l'observation, leur curiosité, leur esprit critique et leur intérêt pour les progrès scientifiques et techniques. "

5• www.lamap.fr

6• www.freinet.org ;

http://fr.wikipedia.org/wiki/Pédagogie_Freinet

7 - Les CCSTI (Centres de culture scientifique, technique et industrielle, www.ccsti.fr/) sont des lieux de médiation scientifique, à destination du grand public, en France. 